\title{
Salud y Enfermedad Mental. Del Corpus Hippocraticum a una aproximación termodinámica
}

\author{
Health and Mental Illness. From Corpus Hippocraticum to a thermodynamic approach \\ Carlos Rojas-Malpica 1,a, Miguel Ángel De Lima-Salas ${ }^{2, b}$, Antonio Eblen-Zajjur ${ }^{3, c}$, Pedro Téllez-Pacheco ${ }^{4, d}$ \\ RESUMEN
}

Se establece una relación entre conceptos básicos acerca de salud y enfermedad mental en los Tratados Hipocráticos y los elementos actuales de la termodinámica y de la cibernética, enlace que se formula a través de los aportes de autores como Maturana y Valera (autopoiesis y teleonomía) o Bergson (regularidades vs indeterminsmos). Se definen y caracterizan los fenómenos anancásticos y los estocásticos, destacándose su relación complementaria. Con un enfoque termodinámico se define a la enfermedad mental como una estructura que se impone hegemónicamente sobre el resto de las actividades mentales y consume la energía que regula el metabolismo restante de la persona afectada. Se aportan evidencias clínicas de las bondades de esta óptica, presentándose, por ejemplo, la ansiedad como una "crisis entrópica" y la depresión como "interferencia de la teleonomía", al tiempo que los trastornos obsesivo-compulsivos son descritos desde una perspectiva cibernética. A lo largo del texto se le confiere mayor relevancia a la noción de la enfermedad mental como "pathos hegemónico" que absorbe recursos energéticos y disminuye las posibilidades de adaptación del afectado a su medio externo y de éxito en el afrontamiento de sus propias tensiones.

PALABRAS CLAVE: Salud y enfermedad mental; epistemología y psiquiatría; medicina hipocrática; termodinámica; cibernética; teleonomía.

\section{SUMMARY}

A link is traced between the basic concepts on health and mental illness in the Hippocratic Treaties and the current elements of thermodynamics and cybernetics, through the contributions of authors such as Maturana and Valera (autopoiesis and teleonomy) or Bergson (regularities vs. indeterminism). Anankastic and stochastic phenomena are defined and characterized, highlighting their complementary connection. Following a thermodynamic approach, a mental illness is defined as a structure that imposes itself on the rest of the mental activities and consumes the energy that regulates the remnants of the affected person's metabolism. Clinical evidences of the benefits of this perspective are given, presenting, for instance, anxiety as "entropic crisis" and depression as an "interference of teleonomy", while obsessive-compulsive disorders are described from the cybernetic perspective. Throughout the

Departamento de Salud Mental. Facultad de Ciencias de la Salud. Universidad de Carabobo. Valencia. Venezuela.

2 Facultad de Medicina. Universidad Central de Venezuela. Caracas, Venezuela.

3 Instituto de Ingeniería Biológica y Médica, Facultades de Ingeniería, Ciencias Biológicas y Medicina, Pontificia Universidad Católica de Chile. Santiago, Chile

4 Hospital Psiquiátrico "Dr. José Ortega Durán”. Valencia. Venezuela

a Profesor Titular, ${ }^{\mathbf{b}}$ Profesor de Historia de la Medicina y de Psiquiatría de Enlace;

c Profesor; ${ }^{\mathrm{d}}$ Director. 
Salud y Enfermedad Mental. Del Corpus Hippocraticum a una aproximación termodinámica.

text, the greatest relevance is conferred to the notion of mental illness as a "hegemonic pathos" that absorbs energy resources and diminishes the affected individuals' possibilities of adaptation to their external environment, and of successfully coping with their own tensions.

KEYWORDS: Mental health and illness; epistemology and psychiatry; hippocratic medicine; thermodynamics; cybernetics; teleonomy.

\section{INTRODUCCIÓN}

La salud y la enfermedad son fenómenos complejos, susceptibles de diversos abordajes y definiciones. Si se contemplan desde la intimidad biológica se llega a una conclusión completamente distinta a la que podría dar lugar una aproximación basada en los componentes ambientales, ecológicos, sociales, o en aquellos más estrictamente personales y subjetivos. Cada perspectiva aporta un ángulo peculiar que puede resultar indispensable para comprender los otros, pero ninguno de ellos funciona por sí solo, sino que forma parte de una red o tejido que se expresa en los diversos estratos biológicos, personales $\mathrm{y} / \mathrm{o}$ sociales de la vida de todo individuo. En psiquiatría es necesario tener presente que pocas veces se trabaja con signos, expresión objetiva de los procesos mórbidos, y sí siempre con síntomas, elementos subjetivos que invariablemente remiten al orden simbólico, a la biografía del sujeto estudiado y sus circunstancias. Es el ser-en-el-mundo heideggeriano incorporado a la clínica, indispensable para un ejercicio profesional significativo.

Desde hace mucho se prefiere hablar del procesosalud-enfermedad (PSE) para insistir en el carácter dinámico, inestable y dialéctico, propio de la relación entre estos dos fenómenos, al tiempo que se enfatiza en un abordaje holístico para resaltar la dimensión global del tema, constituido por elementos inseparables entre sí. Partiendo de tal complejidad, se utilizan en este trabajo, conceptos forjados en la Antigua Grecia, que nunca han perdido vigencia, para interpretar los aportes de la termodinámica, la cibernética, y el pensamiento complejo de Edgar Morín, y así complementar otros discursos sobre el PSE, especialmente en lo que se refiere a detalles de salud y enfermedad mental (1).

\section{Salud y enfermedad en la Antigua Grecia}

La Antigua Grecia otorgó una importancia relevante al problema de la salud y la enfermedad. La naciente Medicina Hipocrática entendió la salud (hygeia) como un estado óptimo de la naturaleza (katá physin), caracterizado por un orden justo de la organización biológica (Dikaios, justo, diké, justicia de la physis), que al mismo tiempo es limpio y puro (katharós), hermoso (Kalós) y proporcionado (metríos). La physiología hipocrática propone que la salud es producto de una predisposición de la naturaleza (diáthesis) y de una mezcla adecuada de sus humores (eukrasia), que deben conjugarse en una relación de proporción y balance (isonomía) en la que el pneuma fluye sin contratiempos (eurróia). Es el desequilibrio de los humores (sangre, flema, bilis amarilla y bilis negra), y el predominio de alguno de ellos (monarkhía) lo que produce la enfermedad (nosos) (2). Destaca aquí la figura de Alcmeón de Crotona (s. VI a.C.) que desarrolló el concepto de «isonomía», equilibrio de cualidades en el cuerpo, y «supremacía», cuando alguna de estas cualidades (seco, húmedo, caliente) "se imponía al resto y producía un desequilibrio, provocando la enfermedad" (3).

Existía también una concepción médica y filosófica del alma, de entrañable y alto valor conceptual para la psiquiatría contemporánea (4). El tratamiento del problema debe ser entendido en el contexto de los ideales de la cultura griega, donde la vida virtuosa o areté, la eudemonia, o estado espiritual que permite una relación armónica con los dioses, la sindéresis (coherencia), la phronesis o control de las pasiones, la verdad (aletheia), la belleza (kalokagatia), así como las virtudes cívicas, fueron valores determinantes de la vida social. La penetración filosófica de los antiguos griegos también permitía distinguir entre opinión (doxa) y conocimiento (episteme) (5). En la Antigua Grecia hubo una armazón conceptual entre medicina y filosofía. La noción de pathos, tanto en epicúreos como estoicos, era entendida como pasión o enfermedad. Para los estoicos, en un primer nivel estaba la euemptosia o predisposición, luego seguía el pathos y al final, la enfermedad propiamente dicha o nosema (6). A todo ello debe añadirse que "el nous como principio ordenador de todo cuanto existe, formulado por Anaxágoras, supondría una naturaleza ordenada de todas las cosas, que siendo asequible al hombre, que como animal de logos, o de orden, podría 
desentrañar tal armonía y restablecerla nuevamente en caso de alteración o predominio" (7). La primera aproximación a la salud y la enfermedad desde una perspectiva científico-natural ocurre en la Antigua Grecia. Sus enseñanzas quedaron vertidas en el Corpus Hipocraticcum, que como es bien conocido, no es una obra de un único autor, sino un compendio de los saberes de su tiempo (Siglos V y IV AC) (8). Las ideas y conceptos hipocráticos se continuaron cultivando en universidades latinoamericanas hasta finales del Siglo XVIII (9).

\section{Algunas precisiones terminológicas y conceptuales}

Los antiguos conceptos griegos de salud y enfermedad han sufrido modificaciones al paso de los siglos y de los avances científicos, de lo cual dan cuenta los diccionarios generales y los terminológicos $(10,11)$. La Organización Mundial de la Salud (OMS) hace tiempo propuso que "La salud es un estado de completo bienestar físico, mental y social, y no solamente la ausencia de afecciones o enfermedades" (12). Para los efectos de nuestra propuesta teórica, interesa destacar que la enfermedad se concibe como una estructura que, según Ferrater Mora, debe ser entendida "como un conjunto o grupo de sistemas. La estructura no es entonces una realidad compuesta de miembros; es un modo de ser de los sistemas, de modo que los sistemas funcionan en virtud de la estructura que tienen" (13). En semiología médica, el síndrome (del griego, $\Sigma v v \delta \rho o \mu \eta$, "concurso") debe ser entendido estructuralmente como un"conjunto de síntomas y signos característicos de una enfermedad o un estado determinado" (14). Conceptos próximos a enfermedad son dolencia, quebranto, malestar, afección, y trastorno, que por lo general son usados para designar alteraciones más leves de la salud.

De Humberto Maturana y Francisco Varela, destacados biólogos y filósofos chilenos, nos interesa

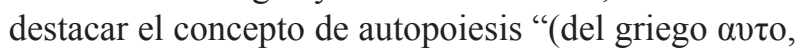

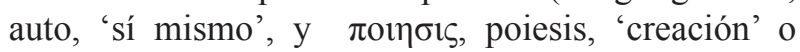
'producción'), un neologismo propuesto en 1971 por Maturana y Varela para designar la organización de los sistemas vivos. Brevemente, podría decirse que la autopoiesis "es la condición de existencia de los seres vivos en la continua producción de sí mismos" (15). Los sistemas autopoiéticos se caracterizan por su autonomía ante el medio ambiente, pues son capaces de hacer surgir un nuevo orden, cualitativamente distinto, a partir de sus componentes moleculares, aún cuando, al mismo tiempo, están sometidos a una "clausura operacional", pues solo pueden dar de sí aquello que está configurado previamente en su estructura genética. Todo ser vivo está en permanente autopoiesis (16). Proponemos contrastar estas propiedades con el concepto de entropía de la segunda ley de la termodinámica, del cual sería su opuesto conceptual.

En relación con la Salud Mental, debemos admitir que no hay consenso para definirla. Bertolote la considera indiferenciable de la salud general: "Cabe destacar que mental, en la definición de salud de la OMS (así como físico y social) se refiere a dimensiones de un estado y no a un dominio o disciplina específicos. En consecuencia, conforme a este concepto, resulta incongruente hablar de salud física, salud mental o salud social". En relación al papel de la psiquiatría “.....dada la naturaleza polisémica de la salud mental, no siempre es evidente su delimitación en relación con la Psiquiatría (entendida como la especialidad médica que se ocupa del estudio, la prevención, el diagnóstico y el tratamiento de trastornos o enfermedades mentales). Hay una fuerte corriente a favor de que, como objetivo mínimo, la salud mental se ubique junto a la Psiquiatría y, como objetivo máximo, que pase a ser un concepto global que incluya a la Psiquiatría..."(17).

\section{Theoría de los desequilibrios}

Los antiguos griegos llamaban $\boldsymbol{\theta} \boldsymbol{\varepsilon} \boldsymbol{c} \boldsymbol{\rho} \boldsymbol{i} \boldsymbol{\alpha}$ (theoría, derivado del verbo theorein) a la actividad mental consistente en contemplar, meditar o especular en abstracto sobre un tema u objeto de estudio. En buena medida, fue así como se desarrolló el Corpus Hippocraticum, aparte de sus amplios contenidos provenientes de la práctica clínica. En este contexto, entendemos como teoría o teorización, a un proceso lógico-deductivo que se propone lograr el máximo de certidumbre posible con respecto a aquello que postula o enuncia, apelando a la heurística, como alternativa para esclarecer y reconocer las lógicas que subyacen al problema de la salud y la enfermedad mental.

Así como los antiguos griegos hablaron de isonomía y harmonía o discordia para referirse al equilibrio o desequilibrio entre los humores y estados de la materia, y de supremacía o monarkhía para identificar el predominio de alguno de ellos, siglos más tarde, Claude Bernard (1813-1868) comenzó a hablar de medio interno (milieu intérieur) para una nueva lectura de los humores, de manera que luego Cannon (1871-1945), inspirado por las formulaciones de Bernard, propuso el concepto de homeostasis. Su contribución fue altamente valorada por Von Bertalannffy (1901-1972) 
Salud y Enfermedad Mental. Del Corpus Hippocraticum a una aproximación termodinámica.

en su "Teoría General de los Sistemas", a la que nos referiremos más adelante. El DTM define homeostasis como "el conjunto de fenómenos de autorregulación de los sistemas biológicos que, en equilibrio dinámico y por mecanismos neurohormonales, tienden a mantener las constantes fisiológicas del medio interno en el organismo frente a los cambios ambientales" (18). Los avances en cronobiología han puesto en evidencia que complejos mecanismos genéticos y de control postranscripcional en realidad no mantienen estables los parámetros fisiológicos, sino que generan oscilaciones en ellos usando como sincronizadores a señales externas como la luz ambiental y la ingesta de alimentos, entre otros (19); esto contrasta con la rígida idea original de homeostasis y genera la necesidad de replantear ambos conceptos, concibiendo la constancia homeostática dentro de las oscilaciones cronofisiológicas.

Hoy también se habla de alostasis para referirse a los factores que desde el medio externo (o medio ambiente) puedan introducir modificaciones en el medio interno. Un abordaje más amplio y contemporáneo no podría ignorar aspectos como los traumas psicológicos, las vivencias tempranas de la vida, las situaciones y conflictos vitales, las enfermedades, los conflictos familiares, laborales, sociales, e incluso las catástrofes, como factores capaces de alterar el equilibrio entre el medio interno y el ambiente. La medicina contemporánea no ignora la subjetividad, los factores sociales, ni tampoco los componentes biológicos del equilibrio. La investigación biológica del estrés ha penetrado en la intimidad de sus mecanismos y en las consecuencias clínicas de la exposición aguda y/o continuada, así como en las posibilidades de intervención terapéutica (20), pero es necesario dejar muy claro que ningún proceso mórbido puede ser entendido prescindiendo de la biografía y de los antecedentes de quien lo padece.

La noción de organismo y de sus equilibrios también puede ser concebida como un sistema abierto. De ninguna manera se puede asimilar a los sistemas adiabáticos de la física, que no establecen relaciones de intercambio de energía con el exterior. Los sistemas biológicos no pueden ser estáticos; por el contrario, están en permanente gestión de sí mismos, como es el caso del embrión que, una vez que comienza su desarrollo, no tiene posibilidad alguna de volver a su estado evolutivo previo. Los sistemas están dotados de un cierto mecanismo teleológico que incorpora lo estocástico, generando múltiples posibles resultados finales. Por otra parte, también pueden ser estudiados desde las perspectivas termodinámica y cibernética $(21,22)$. Según Edgar Morin, entre el sujeto y su ambiente, no hay una separación tajante, sino una relación de autonomía/heteronomía (23). Por su parte, Sigmund Freud planteó el problema como un fragor pulsional entre eros y thanatos: "Esto es favorable a la hipótesis de que el proceso de la vida del individuo conduce, obedeciendo a causas internas, a la nivelación de tensiones químicas; esto es, a la muerte, mientras que la unión con una sustancia animada, individualmente diferente, eleva dichas tensiones y aporta, por decirlo así, nuevas diferencias vitales, que tienen luego que ser agotadas viviéndolas" (24). Pero la vida, en su proceso de gestión y preservación, encuentra alternativas y sumideros para la entropía, que entre los seres humanos puede ser producto de una deliberación socialmente consensuada. Existen jerarquías y procesos generativos que algunos proponen estudiar bajo el concepto de teleonomía que apunta a que el propósito o programa vital del futuro influye y regula los procesos presentes (25).

Henri Bergson (1859-1941), notable filósofo francés, planteaba que el hombre es irreductible a la naturaleza. Se enfrentó vigorosamente a las tesis positivistas y darwinianas que imperaban en su tiempo, a las que calificó de mecanicistas. Para Bergson, la inteligencia y el instinto se dan juntos en las manifestaciones iniciales de la vida, y en el ser humano sufren una escisión radical, para que luego la ciencia, al construir todo su saber con la razón, deje por fuera los impulsos que promueven sus procesos. Por eso la ciencia cruje cuando trata de explicar la vida. En su tesis, por debajo de la inteligencia y el instinto, subyace el élan vital, (concepto introducido por Bergson en su obra La evolución creadora, de 1947, y que se traduce habitualmente como "fuerza o impulso vital) indispensable para comprender la vida (26), propuesta que muy bien podría asociarse a la teleonomía. Sin duda el pensamiento bergsoniano aporta una luz metafísica ausente en otros estudiosos, y es de particular importancia en el modelo de salud mental que nos proponemos presentar en esta comunicación.

¿Cómo se expresan las regularidades y la indeterminación en la intimidad biológica? La vida sana y la enferma se debaten entre novedades azarosas y regularidades indispensables para el equilibrio interior. El medio interno requiere de ciertas estabilidades para conservar las funciones vitales. La concentración de glucosa, de oxígeno, de $\mathrm{CO} 2$, el equilibrio ácido/base y 
las regulaciones hormonales admiten ciertos márgenes de oscilación, por fuera de los cuales fácilmente se perturba la fisiología y se puede comprometer la vida o la salud. Son procesos inconscientes controlados desde el hipotálamo, pero con participación del tallo e incluso de la corteza cerebral: el eje córticohipotalámico-hipofisiario-tiroideo-adrenal-gonadal. Al mismo tiempo, la misma fisiología altera sus equilibrios y constancias, introduciendo caos y azar que ponen a prueba sus estabilidades, como en la fase de movimientos oculares rápidos del sueño, en que se desregula el ritmo cardíaco, la frecuencia respiratoria y se producen fenómenos hípnicos que algunas veces pueden perturbar la tranquilidad del durmiente $\mathrm{y}$ despertarlo en medio de una angustiosa pesadilla o de una placentera experiencia onírica.

El metabolismo y los equilibrios neurohumorales no son estáticos, rígidos, ni inmodificables. La producción hormonal varía con el ritmo circadiano, catamenial y gestacional (incluidos los ritmos infray ultradianos). Decían los antiguos griegos que "el cuerpo del hombre es apomímèsis tou holou, es decir, copia del todo" (27). Igualmente, los ritmos están también presentes en la naturaleza, como ocurre con las cuatro estaciones, como los períodos de lluvia y de sequía, como la noche sigue al día, todos de gran impacto en la vida humana. Las regularidades impuestas por imperiosa necesidad de la naturaleza se denominan fenómenos anancásticos (de Ananké o $A v \alpha \gamma \kappa \eta$, la madre de las Moiras, personificación de lo inevitable, de la necesidad, de la compulsión y de lo ineludible, de lo determinístico); en tanto que aquellos que ocurren de manera azarosa, aleatoria, inadvertida, inesperada, no determinística, se les conoce como

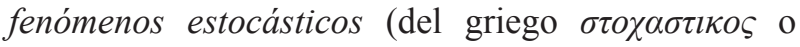
stochastikós, "hábil en conjeturar", relacionado con Thyké, diosa de la fortuna) (28). En condiciones de vida saludable hay equilibrio entre los procesos anancásticos/determinísticos y estocásticos. Ambos conceptos se vinculan con lo discutido en los párrafos anteriores a propósito de los procesos evolutivos y con las ideas propuestas por Bergson. Hay en la filogénesis y en la ontogénesis una tendencia a la complejidad, a la progresiva individuación y a la expresión novedosa de la vida, que pueden ser estudiadas en la evolución del SNC, puntos que también abonan la tesis de este trabajo.

Si se representa la distribución del caos en la naturaleza como una campana de Gauss, se encontraría en el extremo izquierdo a los minerales, siempre iguales a sí mismos y de comportamiento altamente predecible. Los seres unicelulares, mucho más complejos que los minerales, pero aún muy elementales en sus reacciones ante los cambios del ambiente, tienen pocas posibilidades de generar cambios en su interior y mucho menos de introducir modificaciones en su entorno inmediato, de tal manera que sus reacciones pueden predecirse con bastante exactitud ( e.g., cálculo de probabilidades). En la medida en que se asciende en la escala biológica se va incrementando progresivamente el repertorio de posibilidades comportamentales y las interacciones entre el organismo y su ambiente se van tornando cada vez más azarosas o estocásticas. La denominada autopoiesis de Maturana se hace cada vez más evidente. Hacia el medio de la campana de Gauss, se encuentra el comportamiento de los seres humanos, ricos en probabilidades de ajuste, en permanente cambio individual y social, con altas cuotas de creatividad y de complejidad, de tal manera que los procedimientos matemáticos y probabilísticos pueden predecir sus ajustes biológicos más elementales aún cuando se muestran insuficientes cuando se trata de explicar sus niveles más complejos de organización. El itinerario evolutivo que conduce hasta el sujeto más complejo de la filogénesis se va enriqueciendo con incertidumbres.

En el extremo derecho de la curva gaussiana, donde reina el caos más absoluto, incompatible con la vida, pero al mismo tiempo indispensable para su existencia en los renglones que lo preceden, se encuentra el mundo atómico y subatómico, cuyas partículas elementales siguen trayectos y asumen estados de la materia o de la energía absolutamente aleatorios e impredecibles (29). Sin embargo, en la terminología reciente de las ciencias duras o naturales, el término caos denota desorden aparente a nuestros sentidos, pero usando herramientas de análisis no lineal o fractales se descubre el orden subyacente. Estas técnicas han demostrado que los sistemas biológicos saludables muestran conductas y funciones con altos niveles de complejidad y de caos que van reduciéndose en la medida en que un estado patológico se presenta y progresa en gravedad; tal es el caso del control de variables neurovegetativas como el ritmo y amplitud respiratoria y la variabilidad del R-R de los registros electrocardiográficos $(30,31)$.

A su vez, en un primer nivel de organización neurológica, están los reflejos monosinápticos, como el arco reflejo medular, seguidos por las series reflejas temporal y espacialmente coordinadas, también denominadas deflejos, como la deglución y 
Salud y Enfermedad Mental. Del Corpus Hippocraticum a una aproximación termodinámica.

la respiración; los procesos neurohumorales para el control del medio interno y la homeostasis se cumplen sobre todo a nivel diencéfalo-hipofisario, pero uno o dos peldaños más arriba, están las emociones y los sentimientos, muy importantes para esta exposición, que se controlan fundamentalmente desde el sistema límbico, con su respectiva regulación cortical. Se usan los términos "sobre todo" o "fundamentalmente", porque el SNC funciona como un todo, y sólo cuando ocurre una abolición de las funciones mentales superiores, como en los estados comatosos, se cancela total o parcialmente la participación del neocórtex. Es así como el neurocientífico Antonio Damasio aborda el tema, señalando que las emociones precedieron evolutivamente a los sentimientos, al estar constituidas por reacciones simples que promueven sin dificultad la supervivencia de un organismo. (32).

Es muy frecuente que al psiquiatra se le pregunte si odiar, llorar, sufrir, preocuparse, o angustiarse es malo o anormal. La respuesta no puede ser cuantitativa ni moralista. En realidad, el ser humano puede experimentar todas las emociones y sentimientos. El repertorio es muy amplio, aunque es posible que el lenguaje para expresar el malestar supere con creces al usado para referirse al bienestar. Siendo consecuentes con el discurso de los antiguos griegos, pero también con la teoría de sistemas de Bertalanffy y la complejidad moriniana, lo saludable es la complejidad sentimental; y lo mórbido, lo no deseable, es la hegemonía, predominio, monarquía o supremacía de un determinado pathos. Se puede adelantar que dichos estados afectivos -o mejor y más comprehensivamente pathos- son también estructuras que tienen un componente biológico sentido en el cuerpo como una vivencia conmovedora, y un componente inseparable del anterior, que es vivido en la conciencia y expresado como lenguaje. Son, además, sistemas que consumen energía, cuyo predominio o hegemonía puede alterar el medio interno y la homeostasis, y al modificar de tal modo el metabolismo, producir un reacomodo en la distribución de la energía, dando lugar a una vivencia de malestar, dolencia, agotamiento, o de franca enfermedad. En cambio, el equilibrio, la isonomía, el adecuado balance de los humores, se vive como una sensación de bienestar, indispensable para la salud mental. La conducta denominada illness behavior por la terminología anglosajona (33), o comportamiento del enfermo, por la antropología médica de Laín Entralgo (regresión, succión por el cuerpo, dependencia y exaltación del yo) (34), dan igualmente cuenta de la simplificación y pérdida de complejidad a la que conduce la enfermedad, especialmente en sus formas crónicas (35). Pero no se debe perder de vista, que así como el proceso mórbido termina organizando el comportamiento del enfermo, se da también un nuevo ajuste a nivel personal, para lograr un nuevo equilibrio aún cuando sea precario; esto ha sido señalado con mucho acierto por Canguilhem a propósito de ciertos acomodos y estabilidades dentro de la enfermedad (36).

Esta discusión, y los datos aportados, son ya base suficiente para examinar la enfermedad mental como una estructura que, al imponerse hegemónicamente sobre el resto de las funciones mentales y, al consumir los recursos energéticos de control del resto del metabolismo, crea un problema termodinámico. Pacientes con enfermedades mentales como esquizofrenia, depresión o trastorno bipolar muestran con mayor frecuencia que la población general alteraciones como obesidad, diabetes, hipertensión y dislipidemia (37). De hecho, la ansiedad y el miedo extremo o pánico, son situaciones que, con cierta flexibilidad en su óptica, pueden ubicarse dentro de la definición del concepto de dolor, como resultado de una situación real o potencial de lesión, tal y como es aceptado por la Asociación Internacional para el Estudio del Dolor (38). En este contexto se dispara la cadena de eventos y cascadas metabólicas inflamatorias que incluye citocinas, prostaglandinas y neurotransmisores, por solo mencionar algunos (39), todos éstos con efecto sistémico(40) que impacta el metabolismo de neurotransmisores, particularmente la adenosina, que ha sido asociada a enfermedad depresiva y a enfermedad bipolar (41).

\section{Las evidencias clínicas}

Desde los tiempos de Pinel (1745-1826) la enfermedad mental fue planteada como una pérdida de autonomía. En su modelo, basado en las observaciones de Hegel (1770-1831) y utilizando el concepto de alienación, lo que caracteriza a la enfermedad mental es la vulneración de la individualidad y de su autonomía. Llama a la enfermedad mental alienación $o$ enajenación del alma. Hoy podríamos entender la pérdida de libertad del enfermo mental como una falla en la autopoiesis o una obstrucción de la subjetividad (42).

Lanteri Laura ((1930-2004), psiquiatra francés estudioso de la historia y epistemología psiquiátricas, identifica un período caracterizado por la construcción teórica de estructuras clínicas, donde ubica las obras de Kraepelin y de Bleuler. A propósito de Henri Ey, el 
mismo autor examina su "hipótesis órgano-dinámica", de fructíferos resultados para la comprensión de los trastornos mentales. La hipótesis de Ey quizás sea uno de los últimos esfuerzos para lograr una comprensión integral de la enfermedad mental. Las investigaciones y logros de las neurociencias contemporáneas, centradas en localizar alteraciones subyacentes (al modo kraepeliniano), podrían ciertamente encontrar una acogida y poder explicativo superior en el modelo organodinámico de Henri Ey $(43,44)$.

Pasaremos enseguida a revisar algunas de las estructuras clínicas de la psiquiatría desde la postura epistemológica presentada arriba. Más que nosografía o nosotaxia, se intentará un ejercicio nosológico que procure capturar y describir las lógicas que subyacen bajo las correspondientes alteraciones mórbidas.

Trastornos de ansiedad. Constituyen un grupo de estructuras clínicas, cuyo denominador común es la ansiedad o angustia. El ataque de ansiedad o crisis de pánico quizás sea el mejor ejemplo para ilustrar este enfoque teórico. En medio de la crisis quedan subsumidas hasta las nociones de tiempo y espacio. No se vive más que un instante aterrador, donde la amplitud del espacio exterior se percibe amenazante. Toda la energía y los procesos metabólicos quedan al servicio de la crisis. Es la absoluta supremacía o hegemonía de un pathos aterrador, donde no hay ningún acceso al inmenso repertorio de sentimientos y emociones del paciente. La erosión metabólica es tan grande, que al concluir la crisis, el enfermo queda exhausto y sin energías, temeroso de una reanudación del episodio. Es muy frecuente que la crisis se repita en otro momento. La naturaleza cerebral ha quedado organizada para reproducir anancásticamente el fenómeno. El enfermo también organiza su vida alrededor del terror vivido, teme abandonar la casa, quiere estar cerca de una emergencia hospitalaria, abandona sus ocupaciones habituales y suplica a sus familiares que no le dejen solo. Progresivamente pierde la plasticidad y frescura propias del comportamiento saludable.

No sería pues exagerado hablar de una crisis entrópica. En este sentido, hay una activación de las cascadas metabólicas de mediadores proinflamatorios generados durante las crisis de pánico (dolor por daño potencial) que en conjunto incrementan la excitabilidad neuronal espinal y especialmente cortical, dada la acción sistémica de los neuromediadores $(39,40)$. El incremento en la excitabilidad neuronal cortical es el fundamento de los fenómenos de potenciación de largo plazo, con el establecimiento de un mayor número de conectividades interneuronales, activación de genes y expresión de receptores y de canales iónicos excitatorios en las membranas neuronales, mecanismos que gradualmente incrementan la frecuencia e intensidad de respuestas ante estímulos y desencadenantes de intensidad cada vez menor $(39,45)$, condenando al paciente a repetir las crisis. Desde el punto de vista termodinámico, este estado podría corresponder a un aumento de la energía potencial del sistema mórbido y a una progresiva reducción en el umbral de activación (46).

Trastorno por Estrés Postraumático. Esta entidad describe cómo un evento traumático puede tener un impacto grave en la conducta futura. Generalmente es superable, pero no son pocos los que quedan con su comportamiento organizado alrededor de la experiencia vivida en el momento. Los que han visto en riesgo su vida por un atraco a mano armada, secuestro, una catástrofe natural o una agresión violenta de cualquier naturaleza, quedan por lo regular anticipando en el ambiente humano y natural los indicadores de un riesgo similar. El miedo, un sistema de alerta protector de la vida, de allí en adelante se activa intensamente con señales de mínima energía. Fácilmente se llega a una crisis entrópica que subsume en un terror paralizante a la persona que padece el trastorno. Los mecanismos de respuesta ante una lesión real y/o potencial juegan un papel importante en este escenario multifactorial; prueba de ello es la respuesta favorable de estos pacientes a medicaciones que apuntan a reducir o bloquear canales y/o receptores excitatorios de la membrana neuronal o a inhibirla, como es el caso del valproato, de la pregabalina, o de la gabapentina (47).

Trastornos obsesivos. El mejor ejemplo de conductas repetitivas que mineralizan el comportamiento y reducen su complejidad, se encuentra en los trastornos obsesivos. Nuevamente es necesario diferenciar la obsesión clínica de las preocupaciones y pequeñas reiteraciones de la vida cotidiana. Una música que se repite en el pensamiento, pero que no llega a constituirse en un pathos hegemónico de la vida mental, así como cualquier otra preocupación más o menos persistente, que no interfiera ni reduzca la complejidad sentimental ni cognitiva, no representa una alteración de la salud mental. Es el criterio cualitativo fenomenológico el que permite distinguir con más acierto un pathos mórbido de otro que no lo sea. Fue Donath, en 1895, el primero en utilizar el término anancasmus para designar un cuadro que había sido descrito previamente por Thomsen 
Salud y Enfermedad Mental. Del Corpus Hippocraticum a una aproximación termodinámica.

como Estado Obsesivo Idiopático. Luego Schneider propuso hablar de Personalidad Anancástica (48), y finalmente, el término ha quedado reducido a describir los trastornos obsesivos más graves. No hay duda de que, en esas circunstancias, la obsesión confisca casi la totalidad de la conducta. Sin embargo, en este trabajo, el término anancástico no se utiliza en relación a los aspectos visibles en la externalidad de la conducta, sino en su sentido hipocrático antiguo: para describir la regulación forzosa del comportamiento desde un lugar anacrónico de la physis, que gobierna la vida mental con evidente supremacía. Recientes investigaciones traslacionales en modelos animales y respuestas terapéuticas en humanos sugieren fuertemente que en el trastorno obsesivo-compulsivo (TOC), existe una hiperactividad de los circuitos cortico-estriado-tálamo-corticales (CETC) con una actividad incrementada del cíngulo anterior, corteza pre-frontal caudomedial, corteza órbito-frontal y el núcleo caudado. Estas áreas se encuentran de una $\mathrm{u}$ otra forma asociadas a funciones ejecutivas y participan en la evaluación del significado de las ejecuciones. La intensa interconectividad, así como la existencia de múltiples asas de retroalimentación positiva entre estas estructuras encefálicas explica la reiteración de las conductas gatilladas, por leves o aún inexistentes estímulos. No es de extrañar que, ante ésta difícil situación, menos de la mitad de los pacientes responden favorablemente a los tratamientos disponibles (49).

Trastornos afectivo-depresivos. La tristeza no es, por sí misma, patológica. Incluso es uno de los sentimientos más importantes, porque nos permite entrar en relación de empatía y solidaridad con el que sufre. Tampoco es un problema cuantitativo ¿Quién le puede decir a una madre hasta dónde debe sufrir por la pérdida de un hijo? El problema, de nuevo, es cuando se estructura como un pathos hegemónico. La profunda alteración que opera en el enfermo depresivo ha sido magistralmente descrita por Alonso-Fernández en su Modelo Vital Tetradimensional de la Depresión. El humor depresivo, el hundimiento energético, la ritmopatía y la discomunicación, son las cuatro dimensiones sobre las cuales descansa la estructura de la depresión clínica. De cada una de estas dimensiones, derivan en cascada una cantidad de síntomas que no podrían ser entendidos aisladamente (50). En las formas más graves de la enfermedad, cuando el pathos depresivo absorbe casi por completo la vida mental, el enfermo pierde complejidad y libertad. Vive cansado y exhausto, sin energías disponibles para otras realizaciones. El futuro, y los mecanismos biológicos para la teleonomía, están profundamente oscurecidos por un presente donde no tiene cabida la esperanza. La desesperación puede conducir al raptus suicida.

El panorama terapéutico de los enfermos depresivos ha mejorado mucho en las últimas décadas; sin embargo, la posibilidad de una recaída siempre estará presente. La experiencia del desequilibrio queda grabada en algún lugar de su naturaleza y siempre estará disponible para reinstalarse en su vida. Cada episodio depresivo, además de una crisis existencial, es también una crisis entrópica. En los últimos años se ha logrado elevar la eficacia de los tratamientos antidepresivos, gracias a la síntesis de nuevos fármacos con capacidad de aumentar los niveles sinápticos de serotonina, principal neurotransmisor involucrado en los estados depresivos, pero comparativamente ha habido mucho menor avance en el conocimiento de las causas primarias de la enfermedad depresiva.

La investigación traslacional ha sobrepasado el interés del conocimiento sobre el trastorno depresivo, más allá del mero nivel de monoaminas disponibles en el espacio sináptico, para perseguir un modelo más integral, incluyendo factores proinflamatorios, neurotrofinas, reacción inmune, expresión genética y especialmente el ambiente propicio para activar esta última. Existe consenso en torno a que cualquier lesión que afecte el circuito límbico-córtico-estriado-pálidotalámico que incluye la corteza órbito-frontal, corteza prefrontal medial, amígdala, hipocampo, estriado ventromedial, tálamo y pálido ventral, es capaz de generar depresión grave (54). Ha sido reportada consistentemente la caracterización de las alteraciones en estos circuitos haciéndose hincapié en la existencia de una bipolaridad funcional entre estas estructuras: por una parte, el circuito ventral (conformado por la amígdala, la ínsula, el estriado ventral y la parte ventral de cíngulo anterior y corteza prefrontal), con funciones afectivas y de identificación del significado emocional del estímulo, la respuesta emocional y su regulación, estructuras que muestran reducción en su actividad regulatoria. Por otro lado, se encuentra la red dorsal y cognitiva formada por el hipocampo y las regiones dorsales del cíngulo anterior y la corteza prefrontal que muestran funciones de regulación del estado afectivo, las cuales incrementan su sensibilidad en los estados depresivos (52).

Otras manifestaciones clínicas. Hay emociones y sentimientos que tratamos de cultivar porque hacen más grata la vida (53). Pero en la vida sana de la cotidianidad hay multitud de situaciones en las que se 
experimentan sentimientos o emociones desagradables que no tienen, en sí mismas, carácter psicopatológico, y que forman parte del repertorio sentimental complejo del que está dotada nuestra subjetividad. La suspicacia, el temor, la rabia, los celos, la desconfianza, la sospecha y la precaución, por citar sólo algunos, pueden cumplir una función adaptativa y de protección muy importante para preservar la vida y la identidad personal. Pero si una persona termina organizando su vida en la convicción de que hay una trama urdida para perjudicarlo, hacerle daño, destruir su reputación, o aniquilarlo, lo más seguro, salvo evidencia en contrario, es que estamos en presencia de un trastorno delirante, que algunas veces puede venir acompañado de fenómenos alucinatorios. Al igual que en las estructuras anteriores, hay una pérdida de complejidad, porque la subjetividad ha terminado consumida por un pathos hegémonico que absorbe recursos energéticos y posibilidades emergentes de adaptación, haciendo al sujeto altamente predecible, sin las novedades propias del comportamiento saludable (54). Hallazgos experimentales en modelos animales y en pacientes sugieren la existencia de una disregulación en la eficiencia de discriminación señal-ruido. Las señales talámicas hacia la corteza sensorial son anormalmente moduladas por fibras serotoninérgicas provenientes del rafe dorsal. Adicionalmente, existe un disbalance entre el glutamato y el ácido gamma-aminobutírico (GABA) y/o sus receptores de membrana en la corteza cerebral, que contribuyen a la generación de alucinaciones como alteraciones de las descargas neuronales corticales y sincronía de las mismas (55).

No siempre es fácil, desde la fenomenología y la contemplación heurística, precisar el carácter sano y/o enfermo de un determinado comportamiento. Tomemos por ejemplo el caso de los tatuajes y de la cirugía estética para modificar algunos rasgos corporales o fisionómicos. Es un patrón cultural aceptado que una mujer se maquille el rostro, use pintura de labios y de uñas para mejorar su apariencia o atractivo personal. Algunas veces se hace un tatuaje muy discreto para el borde de los labios y de las cejas. Difícilmente un psiquiatra occidental calificaría tales añadidos como patológicos. No restan complejidad ni empobrecen la vida, y pueden mejorar la autoestima. Además, recordemos que para los antiguos griegos, la salud era también Khalos, que significa belleza. Por otra parte, los límites entre los roles de género se hacen cada vez más borrosos e innecesarios. Un varón también puede maquillarse o llevar un arete colgando del lóbulo de la oreja. Un tatuaje discreto puede resultar visualmente grato tanto en el hombre como en la mujer. Es difícil establecer el momento en el que el tatuaje comienza a ser patológico. Algunas personas cubren casi toda su piel con diversos tatuajes indelebles, lo cual puede tomar varios años. Nuevamente, los límites no son cuantitativos. No hay una medida de superficie corporal a partir de la cual se comience a hablar de trastorno o patología.

El problema es aún más delicado en estos tiempos que exigen tolerancia y respeto de y hacia todos. La medicina y la psiquiatría no pueden hablar sobre el tema desde una posición de autoridad. Entonces es necesario penetrar en el significado del proceso, en lo que se expresa a través de la piel. No es posible comprender el problema prescindiendo de la biografía y del estudio de la complejidad existencial, y de allí que lo grabado en la piel sea sólo un síntoma. A partir de allí será posible contemplar si el proceso se ha constituido en una estructura mórbida. Algunas veces la modificación fisionómica adquiere dimensiones verdaderamente preocupantes. Hay quienes se abren ojales en la frente para insertar cuernos extraños, otros quieren extirparse el apéndice nasal para intentar en su rostro la forma de una calavera, y algunos reivindican el derecho, más allá de los transgéneros, a adquirir la apariencia de un animal, es decir, a ser trans-especie. Nada fácil para la psiquiatría del Siglo XXI, una ciencia que aspira y desea promover la libertad (56).

\section{Corolario}

Los conceptos de salud y enfermedad todavía admiten otras aproximaciones teóricas. Además de los criterios estadísticos de normalidad, así como los de la clínica y la salud pública tradicionales, es posible abordar el problema desde la termodinámica y la cibernética, que en nuestro caso, se inspira en conceptos de la tradición hipocrática.

Los conceptos de medio interno, metabolismo y homeostasis, a nuestro juicio inspirados en los de isonomía, humores, monarkhía, khalos, katá physin, eukrasía, anancástico, estocástico y algunos otros de los tratados hipocráticos, lucen indispensables para hablar de las estructuras clínicas de la psiquiatría en términos de la teoría de sistemas de Bertalanffy, la complejidad de Edgar Morín, la filosofía de Bergson, la termodinámica y la cibernética contemporáneas.

La psiquiatría contemporánea ha aceptado la subjetividad como tema nuclear de su preocupación y estudio. Sabe que trabaja poco con signos, evidencias objetivas de alteración orgánica y más con síntomas 
que portan un importante componente simbólico al que sólo es posible acceder por medio de la biografía del paciente, el análisis existencial, la fenomenología o el psicoanálisis. Es por ello que las estructuras mórbidas sólo pueden ser entendidas cuando se inscriben en el ser-en-el-mundo heideggeriano.

Por la vía de la contemplación y de la heurística $(\Theta \varepsilon \omega \rho \imath \alpha)$ de la salud y la enfermedad, hemos planteado que la mayoría de los denominados trastornos mentales son estructuras clínicas y no un simple agregado de síntomas. En nuestro estudio, son pathos hegemónicos, que consumen mucha energía, incluso la sustraen de otros sistemas biológicos del organismo, y al instalarse como núcleo de vida de las personas enfermas, reducen su complejidad, circunscriben el comportamiento a formatos anancásticos altamente predecibles, afectan los mecanismos teleonómicos inscritos en la biología y, en suma, alteran la salud mental, y confiscan la libertad.

Conflicto de Intereses: Los autores declaran que no hay conflicto de intereses relacionado con la realización del presente trabajo

Financiamiento: Los autores declaran que tratándose de un trabajo eminentemente teórico, no han requerido de ninguna fuente de financiamiento externa para su realización.

\section{Correspondencia}

Carlos Rojas-Malpica

Correo electrónico: carlucho2013@gmail.com

\section{REFERENCIAS BIBLIOGRÁFICAS}

1. Guerrero L, León A. Aproximación al concepto de salud. Revisión histórica. Fermentum Revista Venezolana de Sociología y Antropología. 2008;18: 610-633. Disponible en: http://oai.redalyc.org/ articulo.oa?id=70517572010. Fecha de consulta: 29 de julio de 2017.

2. Laín P. Historia de la medicina. Madrid: Masson S.A; 2003.

3. Sierra C. Notas sobre medicina y difusión de ideas en la Grecia clásica. Estudios griegos e indoeuropeos. 2012, 22: 91-101.

4. Kharavatos A, Ploumpidis D, Christodolou G. Introduction. En: Christolodou G, Karavatos A Anthology of Greek Psychiatric Texts. Athens, Greece: Beta Medical publishers, 2011.

5. Jaegger Wr. Paideia. Los ideales de la Cultura Griega. Ciudad de México: Fondo de Cultura Económica;
2004.

6. Foucault M. La hermenéutica del sujeto. Madrid: Ediciones Akal SA;2005.

7. Martínez H. Medicina y sabiduría:Acerca del proceso morboso y de la naturaleza. Franciscanum Revista de las Ciencias del Espíritu. 2006;142:11-38.

8. Ivanovic-Zuvic F. Consideraciones epistemológicas sobre la medicina y las enfermedades mentales en la antigua Grecia. Rev chil neuro-psiquiatr. 2004; 42(3): 163-175. Doi: http://dx.doi.org/10.4067/ S0717-92272004000300002.

9. Tamaríz F. Physiologia Prima Medicinae. Tomada del cuadernillo: Physiologia prima medicinae/ Cuaderno de Medicina, comenzado el día 16 de Marzo de 1796, y perteneciente a/ Jossé Rafael de Jesús Rodríguez/ día 29 o 30/ Leído por el Dr. D. Felipe Tamaríz. Brunni Celli B, Muñoz García A (Eds). Universidad Central de Venezuela. Caracas. 2001.

10. Real Academia Española Diccionario de la Lengua Española. Madrid: Espasa Calpe; 2001.

11. Real Academia Nacional de Medicina. Diccionario de Términos Médicos (DTM). Madrid: Editorial Médica Panamericana; 2012.

12. World Health Organization. Constitución de la Organización Mundial de la Salud. New York: Conferencia Sanitaria Internacional; 19 de junio de1946.

13. Ferrater J. Diccionario de Filosofía. Barcelona: Editorial Ariel S A; 2004.

14. Diccionario Enciclopédico Vox. Enfermedad. Larousse Editorial SL ; 2009.

15. Letelier JC. Los derroteros científicos de Francisco Varela (1946-2001). Biol Res. 2002; 34(2): 7-13.

16. Maturana H, Varela F. De máquinas y seres vivos. Autopoiesis: la organización de lo vivo. Buenos Aires: Editorial Universitaria, Grupo Editorial Lumen; 2003.

17. Bertolote J. Raíces del concepto de salud mental. World Psychiatry. 2008;7:113-116.

18. Real Academia Nacional de Medicina. Diccionario de Términos Médicos (DTM). Madrid: Editorial Médica Panamericana; 2012.

19. Touitou Y, Erhard H, eds. Biologic rhythms in clinical and laboratory medicine. Berlin: Springer Science \& Business Media; 2012.

20. Abdallah C, Lynnette A, Akiki TJ, Krystal JH. The Neurobiology and Pharmacotherapy of Posttraumatic Stress Disorder. Annual Review of Pharmacology. 2018, 59(1):0-0. DOI: 10.1146/ annurev-pharmtox-010818-021701.

21. Von Bertalanffy, L. Perspectivas en la Teoría General de Sistemas. Madrid: Alianza Universidad; 1975.

22. Del Castillo LF, Vera-Cruz, P. Thermodynamic Formulation of Living Systems and Their Evolution. Journal of Modern Physics. 2011; 2: 379-391. 
doi:10.4236/jmp.2011.25047

23. Morin E. El pensamiento Complejo. Barcelona: Editorial Gedisa SA; 2003.

24. Freud S. Obras Completas. Vol I y II. Madrid: Editorial Biblioteca Nueva;1948.

25. Kirmayer L. Ontologies of life: From thermodynamics to teleonomics. Phys Life Rev. 2017; DOI: https:// doi.org/10.1016/j.plrev.2017.11.022

26. Bergson H. La evolución creadora. Madrid: Editorial Aguilar.

27. Laín P. La Medicina Hipocrática. Madrid: Ediciones de la Revista de Occidente; 1970.

28. Rojas-Malpica C. El fenómeno anancástico en psiquiatría. Anclajes y albures de la vida mental. Anales de la Real Academia Nacional de Medicina. 2012; 129:163-178.

29. Rojas C, Gómez-Jarabo G, Villaseñor S. La Enfermedad Mental como ananké. Investigación en Salud. 2004; 6(3): 159-164.

30. Mauo-Ying B. Breathing pattern variability: a weaning predictor in postoperative patients recovering from systemic inflammatory response syndrome. Intensive care medicine. 2004; 30 (2): 241-247.

31. Gospodinov M. Nonlinear analysis of heart rate variability in Type 2 diabetic patients. Fractal Geom Nonlinear Anal Med Biol. 2016; 1(14): 0-0.

32. Damasio A. En busca de Spinoza. Neurobiología de la emoción y los sentimientos. Barcelona Crítica SL; 2007.

33. Mechanic D. The concept of illnes behavior: Culture, situation and personal predisposition. Psychological Medicine.1986;16: 1-7.

34. Laín P. Antropología Médica. Barcelona: Salvat Editores; 1984.

35. Montalvo-Prieto A, Cabrera-Nanclares B, QuiñonesArrieta S. Enfermedad crónica y sufrimiento: revisión de literatura. Aquichan]. 2012; 12(2): 134-143.

36. Caponi S. Georges Canguilhem y el estatuto epistemológico del concepto de salud. História, Ciências, Saúde- Manguinhos. 1997; 4(2): 287-307.

37. De Hert M. Cardiovascular disease and diabetes in people with severe mental illness position statement from the European Psychiatric Association (EPA), supported by the European Association for the Study of Diabetes (EASD) and the European Society of Cardiology (ESC). European psychiatry. 2009; 24(6): 412-424.

38. Merskey H. The taxonomy of pain. Med Clin North Am. 2007;91(1):13-20. DOI: 10.1016/j. mena.2006.10.009

39. Willis J, William D, Coggeshall RE. Sensory mechanisms of the spinal cord: Volume 1 primary afferent neurons and the spinal dorsal horn. Berlin: Springer Science \& Business Media; 2012.
40. Vicci H. Enoxaparin pretreatment effect on local and systemic inflammation biomarkers in the animal burn model. Inflammopharmacology. 2018; 1: 1-9.

41. Chiu G, Freund G. Modulation of neuroimmunity by adenosine and its receptors: metabolism to mental illness. Metabolism. 2014; 63(12): 1491-1498.

42. Pinel F. Tratado médico-filosófico de la enajenación del alma o manía. Madrid: Imprenta Real; 1804.

43. Lanteri-Laura G. Ensayo sobre los paradigmas de la psiquiatría moderna. Madrid: Editorial Triacastela; 2000.

44. Ey H. La Conciencia. Madrid: Editorial Gredos SA; 1967.

45. Goto Y, Grace A. Alterations in medial prefrontal cortical activity and plasticity in rats with disruption of cortical development. Biological psychiatry. 2006; 60(11): 1259-1267.

46. Wilming H, Gallego R, Eisert J. Second law of thermodynamics under control restrictions. Phys Rev E. 2016; 93: 042126.

47. Hoskins M. Pharmacotherapy for post-traumatic stress disorder: systematic review and meta-analysis. The British Journal of Psychiatry. 2015; 206(2): 93100.

48. Vallejo J. Estados obsesivos. Barcelona: Salvat Editores SA; 1987.

49. Monteiro P, Guoping F. Learning from animal models of obsessive-compulsive disorder. Biological psychiatry. 2016; 79 (1): 7-16.

50. Alonso-Fernández F. Las cuatro dimensiones del enfermo depresivo. Madrid: Instituto de España; 2009.

51. Neha J, Steffens DC. Neurobiology and Risk Factors of Late-Life Depression: Understanding Depression. Singapore: Springer; 2018.p. 279-295.

52. Phillips ML, Drevets WC, Rauch SL, Lane R. Neurobiology of emotion perception II: implications for major psychiatric disorders. Biol Psychiatry. 2003;54(5):515-28.

53. Machado L, Cantilino A. A systematic review of the neural correlates of positive emotions. Revista Brasileira de Psiquiatria. 2017;39:172-179.

54. Rojas C. Las alucinaciones y el delirio como representaciones anancásticas. Salud Mental. 2010; 33: 379-387.

55. Robbins TW. Animal models of hallucinations observed through the modern lens, Schizophrenia Bulletin. 2017; 43(1): 24-26. DOI: https://doi. org $/ 10.1093 / \mathrm{schbul} / \mathrm{sbw} 134$

56. Alonso-Fernández F. El hombre libre y sus sombras: Una antropología de la libertad; Los emancipados y los cautivos. Barcelona: Anthropos Editorial; 2006.

Recibido: $28 / 10 / 2018$

Aceptado: 16/12/2019 